\title{
PAPEL DO ENFERMEIRO NO PERÍODO PERIOPERATÓRIO PARA PREVENÇÃO DA TROMBOSE VENOSA PROFUNDA
}

\author{
The role of the nurse along the perioperative period in order to prevent deep vein thrombosis \\ Rol del enfermero en el período perioperatorio para la prevención de la trombosis venosa profunda
}

\author{
Nathália Gustavo Pinho', Karin Viegas², Rita Catalina Aquino Caregnato²
}

RESUMO: Objetivos: Conhecer como os enfermeiros realizam a prevenção da Trombose Venosa Profunda (TVP) em pacientes submetidos a cirurgias de grande porte no período perioperatório; e levantar os fatores de risco para o desenvolvimento de TVP identificados pelos enfermeiros. Método: Estudo de caso com abordagem qualitativa. Amostra composta por 12 enfermeiros da unidade de internação, centro cirúrgico e sala de recuperação pós-anestésica de um hospital especializado em trauma de Porto Alegre (RS). Utilizou-se entrevista semiestruturada com 10 questões norteadoras. Para interpretação dos dados utilizou-se Análise de Conteúdo de Bardin. Resultados: Emergiram três categorias: Fatores de Risco para TVP; Medidas Preventivas de TVP e Dificuldades na Execução da Sistematização da Assistência de Enfermagem Perioperatória. Conclusão: Os enfermeiros realizam prevenção de TVP com massagem, observação, exame físico/avaliação, medidas protetivas e mudança de posição, entretanto falta autonomia para aplicar algumas medidas preventivas. Apontaram vários fatores de risco para a ocorrência de TVP conforme literatura, evidenciando conhecimento sobre a patologia.

Palavras-chave: Trombose venosa. Enfermagem perioperatória. Prevenção de Doenças.

ABSTRACT: Objectives: Determining how nurses implement deep vein thrombosis (DVT) prevention during the perioperative period in patients submitted to large surgeries and detecting the risk factors to the occurrences of DVT previously traced by nurses. Methodology: A case study with a qualitative approach. A sample of 12 nurses in the inpatient unit, operating room, and postanesthesia recovery room of a hospital specializing in trauma Porto Alegre (RS), Brazil. We used semi-structured interviews with 10 leading questions. For data interpretation, the Content Analysis Method introduced by Bardin was adopted. Results: Three final categories came up from the content analysis: risk factors to DVT, preventive measures to DVT, and adversities in implementing the systematization of nursing care in perioperative. Conclusion: Nurses perform prevention of DVT with massage, observation, physical examination/assessment, protective measures, and change of position but lack autonomy to apply some preventive measures. They showed several risk factors for the occurrence of DVT according to the literature, proving they have the knowledge about the disease.

Keywords: Venous thrombosis; Perioperative nursing; Disease Prevention.

RESUMEN: Objetivos: Conocer como los enfermeros realizan la prevención de la trombosis de la vena en los pacientes sometidos a las cirugías de porte grande en el período perioperatorio; y colectar los factores de riesgo para el desarrollo de la trombosis de la vena que fueron identificados por los enfermeros. Método: Estudio del caso con el enfoque cualitativo. La muestra fue compuesta por 12 enfermeros de la unidad de hospitalización, quirófano y sala de recuperación post-anestesia de un hospital especializado en traumas en Porto Alegre, Rio Grande do Sul, Brasil. Se aplicó una entrevista semiestructurada con 10 preguntas principales. Se utilizó, para la interpretación de los datos, el análisis del contenido de Bardin. Resultados: Se emergieron tres categorías: los factores del riesgo para la trombosis de la vena; las medidas preventivas de la trombosis de la vena y las dificultades en la ejecución de del Sistematización de Atención de Enfermaría Perioperatória. Conclusión: Los enfermeros realizan la prevención de la trombosis de la vena con el masaje, la observación, el examen / evaluación física, las medidas de protección y el cambio de posición, pero carecen de autonomía para aplicar algunas medidas preventivas. Ellos mostraron varios factores de riesgo para la aparición de la trombosis de la vena de acuerdo a la literatura, mostrándose el conocimiento sobre la enfermedad. Palabras clave: Trombosis de la vena. Enfermería perioperatoria. Prevención de Enfermedades.

'Enfermeira, graduada pela Universidade Federal de Ciências da Saúde de Porto Alegre (UFCSPA).

${ }^{2}$ Enfermeira, Doutora pela UFCSPA - Porto Alegre (RS), Brasil. E-mail: ritac.ufcspa@gmail.com

Rua Dr. Rodrigues Alves, 273, apto. 203 - Chácara das Pedras - CEP: 91330-240 - Porto Alegre (RS), Brasil.

Recebido: 25 nov. 2015. Aprovado: 19 fev. 2016

DOI: $10.5327 / Z 1414-4425201600010005$ 


\section{INTRODUÇÃO}

O papel fundamental da enfermagem no período perioperatório envolve a promoção, manutenção e recuperação da saúde do paciente submetido a uma cirurgia de grande porte. É importante que o enfermeiro tenha o conhecimento necessário para avaliar o tipo de cirurgia, identificando a probabilidade de ocorrer uma Trombose Venosa Profunda (TVP)․․

Algumas atribuições são específicas do enfermeiro que atua neste serviço, como, por exemplo, supervisionar as ações de enfermagem necessárias para cada paciente, elaborando normas e rotinas para cada área; saber elencar as prioridades de cada paciente; dependendo da rotina do serviço de saúde, o enfermeiro realiza a avaliação pré-operatória do paciente, entre outras ${ }^{2}$.

A Sistematização da Assistência de Enfermagem Perioperatória (SAEP) tem como objetivo desenvolver uma "assistência integral, continuada, participativa, individualizada, documentada e avaliada"”.

Entende-se como período perioperatório o intervalo que abrange o pré-operatório, o transoperatório e o pós-operatórioº ${ }^{2}$.

A TVP é o desenvolvimento de um coágulo dentro de um vaso sanguíneo venoso, decorrente de uma reação inflamatória ou trauma, determinando obstrução total ou parcial do vaso ${ }^{4}$, podendo levar ao Tromboembolismo Venoso (TEV), em que o coágulo se desloca pelo vaso, estando sujeito a desenvolver uma embolia pulmonar (EP), importante causa de morbidade e mortalidade 5 .

O TEV é considerado um problema de saúde pública pois, além de possuir uma alta taxa de mortalidade, afeta grande parte da população hospitalizada. Outro aspecto importante desta complicação é que a maioria das mortes ocasionadas pelo TEV poderia ser evitada; porém, os métodos farmacológicos para sua prevenção são subutilizados ${ }^{6}$. Desde o início do século XX, recomenda-se prevenir o TEV em pacientes cirúrgicos, mas somente nos últimos 25 anos ocorreu uma objetiva preocupação com esta questão e um aprofundamento nas pesquisas e métodos profiláticos ${ }^{4}$.

O paciente antes de ser submetido a um procedimento cirúrgico deve ser avaliado pela equipe quanto aos seus fatores de risco e o tipo de cirurgia que irá realizar, para então determinar o risco de desenvolver TVP $^{7}$.

As diretrizes do American College of Chest Physicians (ACCP) não apenas indicam observar se o paciente possui riscos para desenvolvimento de TVP e TEV como também aconselham que os hospitais criem estratégias identificando esses riscos e, consequentemente, evitando mortalidade ${ }^{8}$. Para isso, é importante avaliar além da idade do paciente, seu estado físico e o porte da cirurgia (maior ou menor); cirurgias consideradas de médio e grande porte, incluindo a intratorácica, intra-abdominal, ortopédica, neurológica, arterial e de risco hemorrágico elevado, possuem maior risco cirúrgico de desenvolver $\mathrm{TEV}^{7}$.

A profilaxia medicamentosa para TEV, apesar de efetiva, não reduz a zero a incidência de TVP pós-operatória. Dados indicam a incidência de TVP pós-operatória nos casos de cirurgia de quadril, quando não realizada a profilaxia, em até $70 \%$ dos pacientes 9 . Torna-se necessário um processo de cuidado e recuperação, com medidas sobre o controle desta complicação. A SAEP é essencial dentro de um ambiente hospitalar para implementar intervenções potencialmente satisfatórias nos pacientes cirúrgicos, considerando os seus fatores de risco, e, consequentemente, para prestar uma melhor assistência ${ }^{10}$.

Não há muitas pesquisas na área de enfermagem identificando o real papel do enfermeiro na prevenção de TVP no período perioperatório das cirurgias de grande porte. Além disto, a avaliação dos registros de intervenções neste cuidado não permitem discutir as ações preventivas realizadas.

Diante do exposto, o presente estudo teve como objetivo geral conhecer como os enfermeiros realizam a prevenção da TVP no período perioperatório em pacientes submetidos a cirurgias de grande porte. O objetivo específico foi levantar os fatores de risco identificados pelos enfermeiros para a ocorrência de TVP.

\section{MÉTODO}

Optou-se por um delineamento de estudo de caso qualitativo, pois este tipo de pesquisa permite interpretar os dados do contexto pesquisado, buscando novas respostas que retratam a realidade, revelando os diferentes pontos de vista sobre o objeto do estudo ${ }^{11}$.

O campo de ação foi um hospital referência no atendimento ao trauma, principalmente de vítimas de acidentes de trânsito, do trabalho, de violência e queimados, de Porto Alegre, Rio Grande do Sul. Este possui 264 leitos para atendimento nas especialidades de traumatologia, ortopedia, bucomaxilofacial, neurocirurgia, queimados, cirurgia plástica, cirurgia do trauma em geral, entre outros. Atende $100 \%$ pelo Sistema Único de Saúde (SUS).

Amostra intencionalmente constituída por 12 enfermeiros, sendo 4 da unidade de internação (UI); 4 do centro cirúrgico 
(CC) e 4 da sala de recuperação pós-anestésica (SRPA) onde permanecem os pacientes de cirurgia de grande porte, nos períodos pré, trans e pós-operatório. Incluiu-se neste estudo os enfermeiros que trabalhavam há um ano ou mais na UI, CC e SRPA do hospital. Excluiu-se deste estudo enfermeiros que não estavam trabalhando no hospital, por estarem em licença médica ou férias, no período da coleta de dados.

A técnica utilizada foi a de amostragem em rede (também denominada de "amostragem bola de neve") ${ }^{12}$. Nesta abordagem, os primeiros indivíduos da amostra indicam outros que atendam aos critérios de inclusão.

Os dados foram coletados por entrevista semiestruturada, com um roteiro dividido em duas partes:

1. dados de identificação do participante, com o intuito de caracterizar os sujeitos. Convencionou-se denominar os sujeitos da pesquisa com a letra "E" que significa "Enfermeiro", seguida do número conforme ordem da entrevista;

2. 10 questões norteadoras para a entrevista, a saber:

1. O que você entende como Sistematização da Assistência de Enfermagem no Perioperatório?;

2. Neste hospital como funciona a Sistematização da Assistência de Enfermagem no Perioperatório de pacientes de cirurgias de grande porte?; 3 .

Cite alguns fatores de risco para a ocorrência de Trombose Venosa Profunda nos pacientes submetidos a cirurgias de grande porte?;

4. Quais medidas você julga importante para a prevenção da TVP?;

5. Você utiliza medidas para prevenir a TVP em pacientes de cirurgias de grande porte? Caso afirmativo, quais são essas medidas?;

6. Como é feito aqui no hospital a prevenção de TVP nos pacientes submetidos a cirurgias de grande porte no pré, intra e pós-operatório?;

7. Você já atendeu pacientes de cirurgias de grande porte que desenvolveram TVP ou TEV?;

8. Caso afirmativo anterior, pergunta-se: Neste paciente foram implementadas medidas preventivas no perioperatório?;

9. Relate um caso que desenvolveu TVP ou TEV;

10. No seu ponto de vista, como você julga que deveria ser realizado a Sistematização da Assistência de Enfermagem no Perioperatório de pacientes de cirurgias de grande porte para prevenção de TVP?

Cada entrevista durou aproximadamente 10 minutos.
Realizou-se um estudo piloto com duas enfermeiras para identificar se a entrevista atendia aos objetivos propostos, não havendo necessidade de mudanças. As entrevistas com os enfermeiros do pré-operatório foram realizadas em uma sala da unidade de internação (UI); com os enfermeiros do transoperatório foram realizados na sala dos enfermeiros do CC; e com os enfermeiros no pós-operatório foram realizadas em uma sala localizada na sala de recuperação pós-anestésica (SRPA).

As entrevistas foram realizadas entre abril e junho de 2015, gravadas em um aparelho celular e transcritas na íntegra, garantindo a veracidade das informações. Para interpretação dos dados, utilizou-se o método de Análise de Conteúdo ${ }^{13}$.

A técnica seguiu as três etapas preconizadas:

1. Pré-análise: realizou-se a transcrição das entrevistas, organização do material, leitura breve dos textos e início da sistematização das ideias para o plano de análise;

2. Descrição analítica: categorização dos dados, separando pelo critério temático, agrupando todos os temas com o mesmo significado;

3. Interpretação inferencial: dados obtidos foram interpretados, desvelados e realizou-se inferências com o objetivo dos resultados tornarem-se válidos e significativos.

A análise foi realizada após leitura, construção do mapa com todas as perguntas e respostas na íntegra. Após leituras e releituras na horizontal e vertical do primeiro mapa, identificaram-se as repetições, construindo-se o segundo mapa, emergindo cinco pré-categorias que foram agrupadas conforme unidade de significados semelhantes. Na terceira e última etapa construiu-se o último mapa com as categorias finais.

O projeto desta pesquisa foi encaminhado via Plataforma Brasil aos Comitês de Ética em Pesquisa da Universidade e do Hospital sendo avaliado e aprovado pelo CEP da Universidade com número do CAAE 39220814.7.0000.5345 e pelo CEP do hospital com número do CAAE 39220814.7.3001.5530.

\section{RESULTADOS}

Quanto ao perfil dos 12 sujeitos pesquisados, 11 eram do sexo feminino; a idade oscilava entre 26 e 59 anos; o tempo de formação profissional variou entre 3 e 35 anos.

$\mathrm{Na}$ análise de conteúdo realizada emergiram três categorias finais: Fatores de Risco para TVP; Medidas Preventivas de TVP e Dificuldades na Execução da Sistematização da Assistência 
de Enfermagem Perioperatória (SAEP). Nestas as unidades de significado semelhantes foram agrupadas e quantificadas pelo número de vezes que se repetiram nas falas dos sujeitos, resultando em subcategorias, conforme apresentado no Quadro 1.

\section{Categoria: fatores de risco para trombose venosa profunda}

Na primeira categoria, denominada "Fatores de Risco para TVP", os participantes apontaram como determinantes da TVP algumas cirurgias, os pacientes idosos e suas comorbidades, imobilidade, obesidade, traumas e fraturas e falta de condutas preventivas. Na subcategoria "Cirurgias" os participantes indicaram aquelas de maior risco para desenvolver a TVP, tais como: quadril; acetábulo; fêmur; tumor cerebral; coluna; plástica; cirurgia longa; cirurgias ortopédicas em geral; cirurgias que complicam e vários tipos de cirurgia concomitantemente.

Quadro 1. Categorias, subcategorias e quantitativo de unidades de registro sobre a Trombose Venosa Profunda. Porto Alegre, 2015.

\begin{tabular}{|c|c|c|}
\hline $\begin{array}{l}\text { Categorias } \\
\text { finais }\end{array}$ & Subcategorias & $\begin{array}{l}\text { Unidades de } \\
\text { Registro }\end{array}$ \\
\hline \multirow{6}{*}{$\begin{array}{l}\text { Fatores de Risco } \\
\text { para TVP }\end{array}$} & Cirurgias & 22 \\
\hline & Idosos e Comorbidades & 20 \\
\hline & Imobilidade & 16 \\
\hline & Obesidade & 7 \\
\hline & Traumas e Fraturas & 5 \\
\hline & $\begin{array}{l}\text { Falta de Condutas } \\
\text { Preventivas }\end{array}$ & 4 \\
\hline \multirow{6}{*}{$\begin{array}{l}\text { Medidas } \\
\text { Preventivas de } \\
\text { TVP }\end{array}$} & Anticoagulação & 31 \\
\hline & Mudança de Posição & 27 \\
\hline & Bota de Retorno Venoso & 24 \\
\hline & Cuidados de Enfermagem & 22 \\
\hline & Garrote Pneumático & 5 \\
\hline & Fisioterapia & 3 \\
\hline \multirow{4}{*}{$\begin{array}{l}\text { Dificuldades na } \\
\text { Execução da SAEP }\end{array}$} & $\begin{array}{l}\text { Inexistência de } \\
\text { Sistematização }\end{array}$ & 16 \\
\hline & $\begin{array}{c}\text { Limitação a Prescrição } \\
\text { Médica }\end{array}$ & 9 \\
\hline & $\begin{array}{c}\text { Condutas Baseadas na } \\
\text { Experiência }\end{array}$ & 6 \\
\hline & $\begin{array}{l}\text { Falta de Conhecimento e } \\
\text { Iniciativa }\end{array}$ & 4 \\
\hline
\end{tabular}

TVP: Trombose Venosa Profunda; SAEP: Sistematização da Assistência de Enfermagem Perioperatória.
Como resposta a uma das perguntas, que solicitava ao sujeito relatar um caso de TVP ou TEV, duas enfermeiras disseram:

Já vi em outros pacientes no hospital cirurgia de quadril que fizeram TVP e já ouvi relatos também de cirurgias de prótese de quadril que o paciente fez tromboembolismo, quase sempre é óbito E5.

[...], mas geralmente são cirurgias que complicam, são as cirurgias trocantéricas, cirurgias de fêmur e idosos. São esses os pacientes que mais tem TVP (E1).

Agrupou-se uma subcategoria "Idosos e Comorbidades", pois geralmente os idosos apresentam outras patologias, decorrentes da idade, as quais passam a ser consideradas comorbidades. Foram citadas como áreas de risco para a TVP: idosos; impossibilidade clínica ou física (distúrbios); pacientes de acidente vascular cerebral (AVC) e comorbidades, como relatam:

Se o paciente já é idoso, tem uma suspeita de alguma coisa, já encaixar nesse pré-hospitalar algum manejo para que evite que ele chegue aqui e desenvolva a TVP (E10).

Nós temos bastante pacientes obesos aqui [...]. Diabetes; pacientes hipertensos; mas são vários fatores [de risco] assim (E9).

Na subcategoria "Imobilidade" foram citados como fatores de risco para a TVP: longa permanência na mesma posição e o posicionamento do paciente.

Então o paciente muito tempo acamado é um paciente que envolve um risco, né, de desenvolver a trombose (E11).

O risco é a permanência na cama, baixa mobilidade e a impossibilidade do paciente em sair da cama. Impossibilidade clínica ou física, enfim. Mas é o tempo que o paciente fica acamado (E4).

"Obesidade" surgiu como quarta subcategoria mais citada quando perguntado aos sujeitos: "Cite alguns fatores de risco para a ocorrência de Trombose Venosa Profunda nos pacientes submetidos a cirurgias de grande porte?", conforme recortes a seguir: 
Obesidade. Paciente que fica muito tempo acamado" (E6); "e ela tinha a questão de ser uma paciente obesa [...] (E9);

a idade é um fator predisponente e a obesidade também, onde há o risco de desenvolver a trombose (E7).

Na subcategoria denominada "Traumas e Fraturas", foram considerados fatores de risco: fraturas grandes; politrauma; fratura de osso longo e pacientes com trauma raquimedular.

Os nossos pacientes de risco que são os TRM (Trauma Raquimedular); pacientes de AVC que ficam paréticos; então são pacientes de alto risco.

Paciente politrauma grave, que teve uma sequela neurológica grave, acamado muito tempo, chegou para nós da UTI com um edema bem importante na perna direita $[\ldots](\mathrm{E} 11)$.

Então na verdade [...], os pacientes ficam acamados por muito tempo, principalmente os pacientes idosos, pacientes com fraturas de osso longos [...] (E3).

Na subcategoria "Falta de Condutas Preventivas" os participantes citaram: não uso de botas de retorno venoso; muita resistência da equipe e recursos humanos deficitários.

Muitas vezes um paciente dependente tem o risco pela própria dependência dele e os recursos humanos que muitas vezes são deficitários (E4).

Também a falta de condutas preventivas, no caso do próprio, nós temos as botas de retorno venoso que seriam para ser usadas durante esse período, mas que tem pouca aceitação por parte da equipe (E1).

\section{Categoria: medidas preventivas de trombose venosa profunda}

Na segunda categoria, denominada "Medidas Preventivas de TVP”, os entrevistados apontaram a anticoagulação, mudança de posição, bota de retorno venoso, cuidados de enfermagem, garrote pneumático e fisioterapia como medidas essenciais para a prevenção da TVP. Na subcategoria
"Anticoagulação" os participantes mencionaram o uso de anticoagulantes no período perioperatório e quando questionados sobre quais as medidas que julgavam importantes para a prevenção de TVP, surgiram os seguintes relatos:

O preparo do paciente no pré-operatório com uso de anticoagulantes sistêmicos intermitentes (E1);

também tem o uso de anticoagulantes no perioperatório (E2).

Na subcategoria "Mudança de Posição" os participantes citaram como medidas preventivas a mobilização das extremidades dos pés; membros elevados; mudança de posição e mobilização precoce.

Estimular a movimentação; deambulação precoce de pacientes que tem condições (E6).

Movimentação do paciente no pós-operatório imediato, quanto o mais precoce possível (E5).

A terceira subcategoria mais citada como importante medida para a prevenção de TVP foi a "Bota de Retorno Venoso". Entretanto, a utilização da bota de retorno venoso está atrelada à prescrição médica, limitando seu uso para a prevenção da TVP.

Eu na sala de recuperação, infelizmente eu só posso atuar se o médico orientar. A enfermeira da sala de recuperação não pode colocar por conta própria uma bota de retorno venoso. Então a bota é colocada com orientação do cirurgião e tem que estar prescrita para ser feita (E1).

Quando o entrevistado é questionado sobre quais medidas ele utiliza para a prevenção de TVP, o mesmo relata:

Botas de retorno venoso somente com prescrição médica (E8).

Na subcategoria "Cuidados de Enfermagem” foram agrupados: orientação do paciente para o pós-operatório; massagem; observação; medidas protetivas para evitar complicações; improvisação nas medidas preventivas; exame físico/avaliação do paciente; meias compressivas e o conhecimento por parte da equipe de enfermagem em relação à prevenção da 
TVP. Quando questionado: "Quais medidas você julga importante para a prevenção de TVP” os entrevistados referiram:

É diariamente fazer uma observação do paciente como um todo; observar se o paciente tem um bom retorno venoso, o pulso pedioso está palpável, se não tem edema em membros inferiores; principalmente em membros inferiores, mas em membro superiores também, analisar se não tem edema, infiltrados; então isso a gente tem que estar bem atento (E11);

conhecimento; tentar fazer com que o paciente mobilize o quanto antes [orientar o paciente no pós-operatório que ele precisa movimentar-se para evitar complicações]. Eu tento assim, avalio, dou uma olhada no geral e procuro questionar a respeito de dor ou alteração de coloração (E10).

A quinta subcategoria, denominada "Garrote Pneumático", mostrou-se como uma das medidas utilizadas para a prevenção de TVP no transoperatório.

Menor tempo de insuflação do garrote pneumático que é um instrumento muito utilizado nas cirurgias da traumatologia ou ortopedia (E5).

No transoperatório é o cuidado com o garrote [pneumático], temperatura e posicionamento do paciente (E7).

A última subcategoria, denominada "Fisioterapia", foi citada por alguns entrevistados quando questionados sobre como é feita a prevenção da TVP nos pacientes submetidos a cirurgias de grande porte:

Pré-operatório é medicamentoso; no transoperatório bota de retorno venoso e no pós-operatório também e depois tem os serviços da fisioterapia (E6).

Na pergunta: "Você utiliza medidas para prevenir a TVP em pacientes de cirurgias de grande porte? Caso afirmativo, quais são essas medidas?”, foi relatado:

Fisioterapia eles fazem também no pós, se eles têm alguma paralisia, hemiplegia, eles já tem direto o atendimento da fisioterapia, que é bem importante para prevenir (E9).

\section{Categoria: dificuldades na execução da sistematização da assistência de enfermagem perioperatória}

Na categoria "Dificuldades na Execução da SAEP" identificou-se a "Inexistência de Sistematização" como a subcategoria mais citada pelos participantes, aparecendo nas falas a necessidade de sua existência; rotinas básicas, sendo definidas algumas ações padronizadas, dependendo da especialidade; planejamento/organização informal difícil de manter, tanto pelos recursos humanos deficitários quanto pelo perfil da instituição (hospital de trauma); não está institucionalizada; necessidade de implementar os diagnósticos de enfermagem com a prescrição dos cuidados. Apresentam-se alguns recortes de falas que exemplificam esta subcategoria:

[...], mas o pós-operatório eu acho que poderia ser feita uma implementação do diagnóstico, a sistematização de fato, implementar a sistematização com a documentação, avaliação das necessidades $[\ldots]$ acho que seria bem importante (E4).

Quando questionado sobre o funcionamento da SAEP, um entrevistado refere:

Não funciona porque não está institucionalizado. Não há sistematização no perioperatório. É realizado uns cuidados de rotina de unidade, e no transoperatório não é realizado até pela baixa quantidade de enfermeiros para realizar este tipo de cuidado (E5).

A falta de recursos humanos é um fator que interfere na implementação da SAEP, além do perfil do hospital ser um aspecto dificultador:

Olha, eu diria que quando a gente olha a escala cirúrgica a gente faz um planejamento, mas ela é bem complicada de ser mantida, por trabalhar em um pronto socorro, e faz com que tenha muita alteração no decorrer [...] nem sempre tudo que tu planeja consegue cumprir (E2). 
A segunda subcategoria mais citada foi a "Limitação à Prescrição Médica", onde os enfermeiros referiram dificuldades em planejar a assistência de enfermagem para realizar as medidas preventivas da TVP porque dependem da prescrição médica. São ações simples, que o profissional de enfermagem conhece e sabe da importância em realizar, mas para as quais não possui autonomia e, de certa forma, sua não implementação acarreta risco para o paciente, como exemplificam:

No pré-operatório, mesmo sendo menor o índice de trombose, também são feitas medidas para prevenir; se o paciente não pode sair da cama, faz fisioterapia igual, mas tudo a gente precisa da liberação do médico [...]. Essa paciente não podia sair da cama, ela não tinha liberação médica para sair da cama, então ai tu já não tem aquela coisa de poder tirar o paciente da cama, então é bem mais difícil de tu fazer essa prevenção (E9).

A gente aqui é limitado às prescrições [se referindo às prescrições médicas]; mesmo com a enfermagem a gente cuida do decúbito, os posicionamentos; porém o uso das botas pneumáticas está vinculado à prescrição [médica] e uso de heparina também (E12).

Esta limitação da enfermagem no perioperatório dificulta a implementação da SAEP e afeta diretamente a prevenção de TVP:

E a enfermagem ter mais autonomia nesse sentido, porque eu vejo muito estar atrelado a prescrição médica; então tem uma mudança de visão que precisa ser valorizada (E10).

As "Condutas Baseadas na Experiência" mostraram ser algo relevante para a dificuldade na execução da SAEP.

Nós temos algumas condutas que são rotinas padrões para cada especialidade. Essa Sistematização foi feita baseada pela nossa experiência e não em bibliografia ou condutas de literatura; somos nós mesmos quem fabricamos as nossas rotinas [...] não tem uma sistematização muito equilibrada, vai do momento de cada paciente (E1).

Quando questionado sobre o funcionamento da SAEP em cirurgias de grande porte, um participante respondeu:
Não temos; a coisa é individualizada de acordo com o profissional que está fazendo a assistência imediata naquele momento; não tem nada escrito, seguimento, com protocolo, nada (E12).

A última subcategoria, denominada "Falta de Conhecimento e de Iniciativa", demonstra o quanto os profissionais não desenvolvem o olhar crítico diante do paciente, muitas vezes focando apenas na patologia e esquecendo de estabelecer o raciocínio clínico diante de outras sintomatologias que ocorrem simultaneamente.

Às vezes um pouco de desconhecimento da própria equipe, que não presta atenção nos fatores e só vai se dar conta quando o paciente já está apresentando a sintomatologia (E10).

[...] Eu acho que falta um pouco mais também, não só de liberdade, mas também de iniciativa para as pessoas da enfermagem ficarem mais ligadas nos sintomas, nas intercorrências que podem acontecer (E1).

\section{DISCUSSÃO}

Nos fatores de risco para TVP os sujeitos entrevistados apontaram a imobilidade, a idade, as cirurgias ortopédicas como a de fêmur, entre outras, da mesma forma como foram citados em outro estudo ${ }^{8}$ que apontou maior risco de desenvolver TVP os pacientes acamados, idosos e submetidos à cirurgia de fratura de fêmur.

No CC, o enfermeiro lida com mais de uma equipe médica; portanto, é importante uma interação entre os profissionais com a finalidade de desenvolver o trabalho de maneira eficiente e efica $z^{14}$. Esta pesquisa constatou dificuldade em realizar a conduta preventiva para a TVP devido à resistência da equipe médica em prescrever certas medidas preventivas eficazes; assim, se torna necessária uma melhor comunicação entre a enfermagem e a equipe médica que atua no CC, objetivando trocar experiências em relação ao paciente, planejando de forma interdisciplinar a conduta preventiva.

A Sociedade Brasileira de Angiologia e Cirurgia Vascular ${ }^{15}$, nas Normas de orientação clínica para prevenção, diagnóstico e o tratamento da TVP, cita como fatores de risco: a obesidade, o trauma, o tempo de cirurgias, dentre outros. 
Os participantes entrevistados citaram obesidade, traumas e fraturas como fatores de risco; entretanto, não citaram as varizes, anestesia geral e gravidez, também fatores de riscos apontados pela Sociedade.

A fratura de fêmur aumenta o risco de TEV; os pacientes com fratura de fêmur apresentaram maior incidência de EP, provavelmente também em consequência da imobilidade prolongada 8 .

Em relação às medidas preventivas de TVP, os participantes apontaram a mudança de decúbito para prevenir a TVP, revelando a importância em movimentar o paciente o mais precocemente possível, conforme cada caso. Um recente estudo $^{8}$ mostrou que a incidência de EP (uma das complicações mais graves da TVP) foi maior nos pacientes acamados se comparados com os não acamados. A mudança de posição do paciente cirúrgico é uma medida que compete à enfermagem realizar, com o intuito de prevenir não só lesões de pele como também TVP $^{3}$.

A Sociedade Brasileira de Angiologia e Cirurgia Vascular ${ }^{15}$ reforça a importância do uso de anticoagulantes para qualquer tipo de cirurgia. A anticoagulação foi a medida preventiva mais citada pelos participantes, ressaltando a importância do uso de anticoagulantes antes e após as cirurgias de grande porte.

A utilização de bota de retorno venoso e as meias compressivas no paciente cirúrgico, no intraoperatório e pós-operatório, mostram-se eficazes no que diz respeito à prevenção de TVP, visto que auxiliam o fluxo sanguíneo e, consequentemente, diminuem a estase venosa, um dos fatores de risco para o desenvolvimento da trombose $\mathrm{e}^{16-17}$. Alguns participantes da pesquisa citaram o uso de meias compressivas quando possível, caso não tenha contraindicação para determinado paciente. O garrote pneumático é um equipamento utilizado pelas equipes nas cirurgias da ortopedia e traumatologia. Nesta medida preventiva a enfermagem não pode intervir, pois é a equipe médica que decide realizá-la no intraoperatório.

Em relação às dificuldades na execução da SAEP, os participantes apontaram a falta de recursos humanos para poder realizar uma assistência adequada, avaliada e documentada. A falta de conhecimento e iniciativa por parte dos profissionais influencia na não adesão da sistematização. Outro estudo realizado menciona os fatores de risco citados anteriormente, porém afirma que o enfermeiro se foca mais em atividades administrativas do que nas assistenciais e, por falta de clareza em relação à SAE, acaba perdendo muito tempo na elaboração do plano de atividades ${ }^{18}$.
O presente estudo corrobora com a literatura no que diz respeito à dificuldade da implementação da SAEP devido ao número reduzido de enfermeiros a realizá-la ${ }^{3}$. Na prática, observa-se que a SAEP está sendo realizada parcialmente, havendo necessidade de implementar uma documentação, com os planejamentos dos cuidados $^{19}$. Outro fator que influencia na SAEP é o fato do perfil do hospital ser um pronto socorro de trauma, geralmente com atuação de mais de uma especialidade, no qual as salas têm que estar preparadas para as eventuais complicações, dependendo muito da evolução dos pacientes, não tendo uma sistematização específica ${ }^{20}$.

Sendo o conhecimento e a capacitação da equipe de enfermagem fatores que podem evitar complicações no pós-operatório ${ }^{3}$, a enfermagem deve conhecer e estar atenta para os possíveis agravos da cirurgia, avaliando individualmente cada paciente para prevenir a TVP.

\section{CONSIDERAÇÕES FINAIS}

Esta pesquisa permitiu conhecer como os enfermeiros de um hospital de referência no trauma realizam a prevenção da TVP no período perioperatório de pacientes submetidos a cirurgias de grande porte. Os cuidados de enfermagem citados para prevenção de TVP no período perioperatório foram: massagem; observação; exame físico/avaliação do paciente; improvisação nas medidas protetivas; mudança de posição. Constatou-se nas falas dos participantes falta de autonomia para o enfermeiro aplicar algumas medidas preventivas, tais como: uso de bota de retorno venoso e meias compressivas, uma vez que a utilização destas está condicionada à prescrição médica. Este fato interfere em uma das etapas do SAEP, pois não é possível planejar uma assistência integral. Além disto, existe deficiência de recursos humanos e falta de conhecimento por parte da equipe em relação ao processo de sistematização da assistência, dificultando a execução da SAEP.

Os enfermeiros apontaram vários fatores de risco para a ocorrência de TVP no perioperatório conforme apresenta a literatura, evidenciando conhecimento sobre a patologia; entretanto, varizes e gravidez não foram citadas.

É importante que o enfermeiro tenha conhecimento, habilidade e atitude para o seu empoderamento, exercendo seu papel frente à equipe de saúde, modificando a prática existente, ainda muito centrada no modelo biomédico. 


\section{REFERÊNCIAS}

1. Luvisotto MM, Vasconcelos AC, Sciarpa LC, Carvalho R. Atividades assistenciais e administrativas do enfermeiro na clínica médicocirúrgica. Einstein. 2010;8(2):209-14.

2. Associação Brasileira de Enfermeiros de Centro Cirúrgico, Recuperação Anestésica e Centro de Material de Esterilização. Práticas recomendadas SOBECC. 6. ed. São Paulo: SOBECC; 2013. p. 175.

3. Fonseca RMP, Peniche ACG. Enfermagem em centro cirúrgico: trinta anos após criação do Sistema de Assistência de Enfermagem Perioperatória. Acta Paul Enferm. 2009;22(4):428-33.

4. Barros-Sena MA, Genestra M. Profilaxia da trombose venosa profunda em pós-operatório de cirurgias ortopédicas em um hospital de traumato-ortopedia. Rev Bras Hematol Hemoter: 2008;30(1):29-35.

5. White RT. The epidemiology of venous thromboembolism. Circulation. 2003;107(23 Suppl 1):14-8.

6. Maynard G, Stein J. Designing and implementing effective venous thromboembolism prevention protocols: lessons from collaborative efforts. J Thromb Thrombolysis. 2010;29:159-66.

7. Fernandes EO, Guerra EE, Pitrez FAB, Fernandes FM, Rosito GBA, Gonzáles HE et al. Avaliação pré-operatória e cuidados em cirurgia eletiva: recomendações baseadas em evidências. Rev AMRIGS. 2010;54(2):240-58.

8. Akpinar EE, Hosgün D, Akan B, Ates C, Gülhan M. A tromboprofilaxia evita o tromboembolismo venoso após cirurgia ortopédica de grande porte?. J Bras Pneumol. 2013;39(3):280-6.

9. Geerts WH, Bergqvist D, Pineo GF, Heit JA, Samama CM, Lassen MR, et al. Prevention of venous thromboembolism. Chest. 2008;133(6 Suppl):381S-453S.
10. Zanchettin SD, Garlet MS, Oliveira VA, Hermel PP, Amestoy SC. Trombose venosa profunda e a importância da sistematização da assistência de enfermagem. In: XX Congresso de Iniciação Científica UFPEL; 2011.

11. Ventura MM. 0 estudo de caso como modalidade de pesquisa. Rev SOCERJ. 2007;20(5):383-6.

12. Polit DF, Beck CT. Fundamentos de pesquisa em enfermagem. 7. ed. Porto Alegre: Artmed; 2011, p. 669.

13. Bardin L. Análise de conteúdo. São Paulo: Edições 70; 2011, p. 279.

14. Stum EMF, Maçalai RT, Kirchner RM. Dificuldades enfrentadas por enfermeiros em um Centro Cirúrgico. Text Contexto Enferm. 2006;15(3):464-41.

15. Sociedade Brasileira de Angiologia e Cirurgia Vascular. Normas de orientação clínica para prevenção, diagnóstico e tratamento da trombose venosa profunda. J Vasc Bras. 2005;4(3):S205-20.

16. Leme LEG, Sguizzatto GT. Profilaxia do tromboembolismo venoso em cirurgia ortopédica. Rev Bras Ortop. 2012;47(6):685-93.

17. Penha GS, Damiano AP, Carvalho T, Lain V, Serafim JD. Mobilização precoce na fase aguda da trombose venosa profunda de membros inferiores. J Vasc Bras. 2009;8(1):77-85.

18. Adamy EK, Tosatti M. Sistematização da assistência de enfermagem no período perioperatório: visão da equipe de enfermagem. Rev Enferm UFSM. 2012;2(2):300-10.

19. Saragiotto IRA, Tramontini CC. Sistematização da assistência de enfermagem perioperatória: estratégias utilizadas por enfermeiros para sua aplicação. Cienc Cuid Saude. 2009;8(3):366-71.

20. Bogdanovic J, Perry J, Guggenheim M, Manser T. Adaptive coordination in surgical teams: an interview study. BMC Health Serv Res. 2015;15:128. 